\title{
$q$-DISCRETE PAINLEVÉ EQUATIONS: THEIR HIERARCHIES AND PROPERTIES
}

\author{
HUDA DAEFALLH ALRASHDI
}

\author{
(Received 1 June 2020; first published online 14 September 2020)
}

2020 Mathematics subject classification: primary 34M55; secondary 33E17, 34K17, 37K10.

Keywords and phrases: q-discrete Painlevé equation, Lax pair, hierarchies, Bäcklund transformation, symmetry group.

The main objective of this thesis is to derive hierarchies of $q$-discrete Painlevé equations. Some of the important properties of these hierarchies are also given, namely Lax pairs, Bäcklund transformations and solutions of their associated linear problems for special values of parameters and their symmetry groups.

To construct these hierarchies, we apply a geometric reduction and a staircase method on a multi-parameter generalised lattice modified Korteweg-de Vries equation. In addition, the property of consistency around the cube is used in order to find Bäcklund transformations.

Starting with the base case of $q$-discrete second, third and fourth Painlevé equations on an $\mathcal{A}_{5}$ initial-values surface, new hierarchies of $q$-discrete third and fourth Painlevé equations are discovered, and we also rediscover the hierarchy of the $q$-discrete second Painlevé equation. In this thesis, we provide the Lax pairs for each member in these hierarchies. Using the consistency around the cube, we also provide the Bäcklund transformation for the entire hierarchy of $q$-discrete second and third Painlevé hierarchies. We generate a hierarchy of special solutions starting with seed solutions for $q$-discrete second and third Painlevé hierarchies.

An assumption made is that particular parameter values would enable the ability to diagonalise the Lax pair. As a consequence we found that the associated linear problem for the three hierarchies can be solved in terms of the $q$-gamma function. Furthermore, the hierarchy of the $q$-discrete fourth Painlevé equation can be reduced to one equation that can be linearised to become a Riccati equation which has hypergeometric special solutions.

Thesis submitted to the University of Sydney in June 2019; degree approved on 4 November 2019; supervisor Nalini Joshi.

(C) 2020 Australian Mathematical Publishing Association Inc. 
Finally, we investigate the affine Weyl group structure of the symmetry group for each hierarchy. We construct the explicit representation of the symmetry group for the first and second member of these hierarchies.

The collection of new hierarchies, their Lax pairs, Bäcklund transformations, the resultant symmetry groups and special solutions comprise the new results of this thesis. The thesis contains material published in [1] in collaboration with N. Joshi and D. Tran. The material of this paper is presented in Chapter 3, and is related to $q \mathrm{P}_{\mathrm{II}}$ and $q \mathrm{P}_{\text {III }}$ hierarchies, their Lax pair and examples. Chapter 4, on Bäcklund transformation of $q \mathrm{P}_{\mathrm{II}}$ and $q \mathrm{P}_{\mathrm{III}}$ hierarchies, also includes material from the above-mentioned paper. Similarly, Chapter 5 reports on results about solutions of the linear problem from the above paper. However, we emphasise that all the results about $q \mathrm{P}_{\mathrm{IV}}$ throughout the thesis are completely new and unpublished. Chapter 6 includes unpublished material even for $q \mathrm{P}_{\text {II }}$ and $q \mathrm{P}_{\text {III }}$ hierarchies.

\section{Reference}

[1] H. Alrashdi, N. Joshi and D. Tran, 'Hierarchies of $q$-discrete Painlevé equations', J. Nonlinear Math. Phys. 27(3) (2020), 453-477.

HUDA DAEFALLH ALRASHDI, School of Mathematics,

King Saud University, Riyadh 11451, Saudi Arabia

e-mail: halrashidi@ksu.edu.sa 